\title{
Using Process Assessment Models based on multiple Process Reference Models in a Capability Determination Context
}

\author{
Terry Rout \\ Software Quality Institute, Griffith University, Queensland, Australia \\ T.Rout@griffith.edu.au
}

\begin{abstract}
Two cases are presented where the use of Process Assessment Models based on multiple Process Reference Models was found to be successful. It is suggested that this approach can be of particular help when the purpose of the assessment involves the determination of process-related risk - Process Capability Determination..

Keywords: Process assessment, ISO/IEC 15504, Process capability determination
\end{abstract}

\section{Introduction}

ISO/IEC 15504 [1] defines an approach to Process Assessment where the capability of processes implemented in an organization is assessed by comparing objective evidence collected in the organization to indicators of performance and capability specified in a Process Assessment Model. The Process Assessment Model is based on an appropriate Process Reference Model, which contains the definitions of the process entities to be assessed, in combination with a specified framework for measurement of process capability, based on a series of defined Levels of Capability.

The Standard contains provisions for Process Assessment Models to be based upon multiple different Process Reference Models, but there have been few reports on the application of this flexibility. This report provides information on the development of an Assessment Model based on multiple Reference Models, and its application in the context of Process Capability Determination [2].

\section{Case Study}

The context for this assessment was the determination of comparative risks associated with two different options for the development and delivery of a suite of services in a large government organization. Units within the organization were involved with both development and delivery of the services, and it was clear that a simple assessment of service management capability alone would not suffice; in addition, it became clear, in the initial scoping of the exercise, that governance issues 
were of considerable importance, and were not adequately covered by the process models most commonly available.

Following detailed discussions with the sponsoring organization, and with representatives of the units subject to the assessment, the Process Scope for the assessment was determined. It was decided initially to combine processes from two available Assessment Models - ISO/IEC 15504-5 (Software Life Cycle Processes) [3], and the Committee Draft version of ISO/IEC 15504-8 (IT Service Management) [5]. Having regard to the management practices in the organization to be assessed, it was decided that the bulk of the management and support processes to be evaluated would be drawn from ISO/IEC 15504-5. However, the processes for budgeting and business relationship management were taken from ISO/IEC 15504-8. Core engineering processes came form ISO/IEC 15504-5; fundamental service management processes from ISO/IEC 15504-8. The process scope initially determined for the assessment thus comprised the following processes:

ISO/IEC 15504-5

ENG.1 Requirements elicitation

ENG.3 Systems architectural design

ENG.9 Systems integration

ENG.10 Systems testing

SPL.3 Product acceptance

MAN.3 Project management

MAN.5 Risk management

SUP. 8 Configuration management

SUP.9 Problem resolution management

SUP.10 Change request management

ISO/IEC 15504-8 (PDTR)

5.2 Budgeting and accounting of IT services

5.3 Business relationship management

5.9 Incident management and request fulfillment

5.16 Release and deployment management

5.24 Service transition

Review of this draft scope with the organization identified the issue (mentioned above) relating to the need to address overall governance. This was resolved by selecting a suitable process from the Val IT model - Investment Management [6]. It was necessary to construct a Reference Model definition for this process from the content of the Val IT document, and to identify assessment indicators consistent with those employed in the ISO/IEC 15504 models. The full process scope for the assessment this covered 16 processes, drawn from 3 different process models.

The Capability Dimension scope for the assessment was determined by examining the levels of potential risk broadly across the organization. Major management 
processes were scoped to Capability Level 3; the remainder of the scope was to Capability Level 2.

The assessment was conducted following the requirements of ISO/IEC 15504-2, and ratings of Process Attribute Achievement were determined for all processes. There were few significant issues in the conduct of the assessment, as the wording of the Practice Indicators were generally consistent. Managing the Work Product indicators was more complex, as the classification scheme was different in the two principal models (15504-5 and 15504-8); the complexity was however manageable.

Application of the Capability Determination approach defined in ISO/IEC 15504-4 proved highly successful in identifying potential risks associated with the two proposed strategies, and enabled clear and justified recommendations to be provided. The overall results of the assessment were regarded as highly successful by the Sponsor.

\section{Further Application}

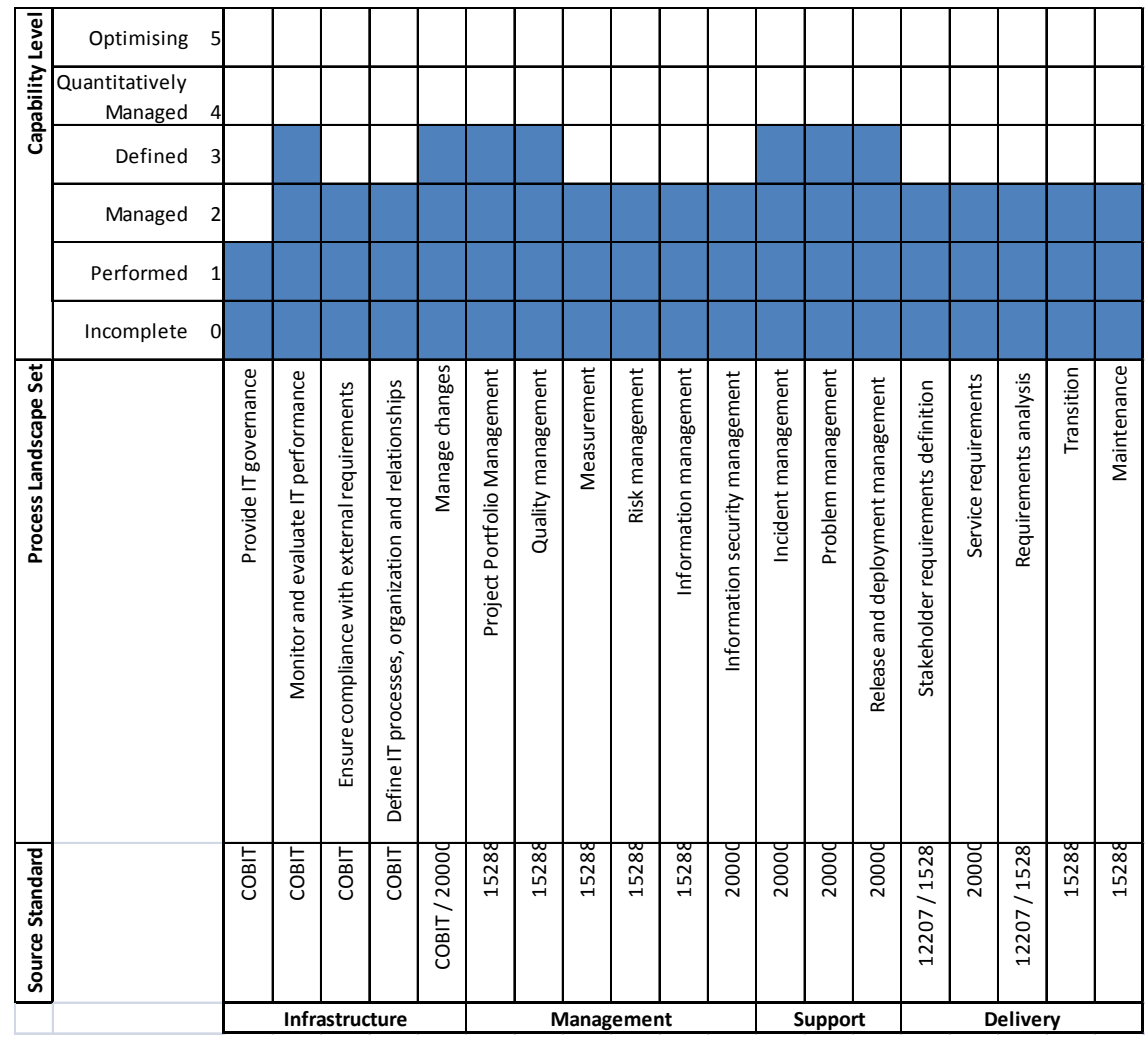

Figure 1 - Target Process Profile, Organization B

Since the conduct of this exercise, a draft Process Assessment Model has become available as part of COBIT V4.1 [7]. In a separate exercise to the principal one 
described above, a Target Profile was determined for risk management purposes in another organization; on this occasion, processes from four separate models were chosen - System Life Cycle processes (drawn from ISO/IEC 15504-6 [4]) were seen as relevant, and COBIT replaced Val IT as a source of governance content. A target profile covering 19 processes was defined, and is shown above, in Figure 1.

At this stage, the assessment for this organization has not been completed; however, the determination of Process Scope again showed the value, in addressing the Sponsor's concerns, of being able to employ multiple different process models.

It is suggested that in the revision of ISO/IEC 15504 (as ISO/IEC 330xx) the assessment framework should facilitate the use of multiple models in a single assessment, particularly where the determination of process-related risk is an aim for the assessment.

\section{$4 \quad$ References}

1. ISO/IEC 15504-2: 2003, Information technology - Process assessment - Part 2: Performing an assessment.

2. ISO/IEC 15504-4: 2004, Information technology - Process assessment - Part 4: Guidance on use for process improvement and process capability determination

3. ISO/IEC 15504-5: 2008, Information technology - Process assessment - Part 5: An exemplar Process Assessment Model

4. ISO/IEC 15504-6: 2008, Information technology - Process assessment - Part 6: An exemplar system life cycle process assessment model

5. ISO/IEC 15504-8: 2008, Information technology - Process assessment - Part 8: An exemplar process assessment model for IT service management

6. IT Governance Institute (2008), Enterprise value: Governance of IT Investments - The Val IT Framework 2.0.

7. ISACA (2011), COBIT Process Assessment Model (PAM), Using COBIT 4.1. 